\title{
A SUFFICIENT CONDITION FOR THE EXISTENCE OF MULTIPLE PERIODIC SOLUTIONS OF DIFFERENTIAL INCLUSIONS
}

\author{
RALF BADER \\ Mathematisches Institut der Universität München, \\ Theresienstraße 39, D-80333 München, Germany
}

1. Introduction. Let $K$ be a convex cone in $\mathbb{R}^{n}$ and $\Phi:[0, T] \times K \rightarrow \mathbb{R}^{n}$ a set-valued map. We shall be concerned with the existence of solutions of the periodic problem

$$
\left\{\begin{array}{l}
x^{\prime}(t) \in \Phi(t, x(t)) \\
x(0)=x(T)
\end{array}\right.
$$

It is known that under regularity conditions on $\Phi$ and a certain Nagumo-type boundary condition a solution of (1) exists (see e.g. [2]).

In this note, following ideas of [4] and using results from [11], we prove the existence of two or more periodic trajectories if additional conditions are satisfied: Our method requires that certain complementary potential functions (comp. [9], [7]) in phase space can be detected.

In our approach we apply the topological fixed point index theory to the Poincaré (also called Poincaré-Andronov) translation operator associated with (1). This well-known technique was developed by M. A. Krasnosel'skiǔ in the single valued case, i.e. when we have unique solvability of the respective initial value problem (see [9]). For applications of the set-valued Poincaré map we recommend [4] as a good survey.

We shall proceed as follows: In section 2 we present the fixed point index theory for the so-called decomposable mappings (see our definition (2.3), comp. [4]) as it is developed in [1] and obtain some additional information necessary for our later work. In the third section we prove some abstract multiple fixed point results for compact decomposable mappings defined on cones of arbitrary ordered normed spaces. We choose this general setting since we believe that the results given there might be of independent interest. Our methods are somewhat connected with the theory given in [10] (for single valued maps), where applications to certain boundary value problems and Hammerstein integral

1991 Mathematics Subject Classification: Primary 34A60; Secondary 58F22.

The paper is in final form and no version of it will be published elsewhere. 
operators are given. Finally in section 4 we state our main results on the existence of periodic solutions.

We conclude the introduction with some notational conventions and definitions.

By a space we always mean a metric space. If $X$ is a space, $A \subset X$ and $\varepsilon>0$, then by $\bar{A}$ and $\partial A$ we denote closure and boundary of the set $A$ respectively and by $N_{\varepsilon}(A)$ we denote an open $\varepsilon$-neighborhood of $A$. By a map we mean a continuous transformation of spaces and by a set-valued map - an upper semi-continuous multi-valued map with compact values. For maps we reserve Latin letters: $f, g, h, \ldots$ and for set-valued maps, Greek letters: $\varphi, \psi, \chi, \ldots$ etc. We say that a set-valued map $\varphi: X \rightarrow Y$ is compact if the set $\overline{\varphi(X)}$ is compact in $Y$ and it is completely continuous if it maps bounded sets onto relatively compact ones.

2. The fixed point index. The main tool in our consideration stand the topological fixed point index as it is introduced in [5] and developed in [1]. All the proofs which are not included here are given in [1]. We would like to mention that this fixed point index can be constructed by using the technique of single valued approximation on the graph.

We consider maps having the so-called proximally $\infty$-connected values. After [3] we recall this notion.

Definition 2.1. A compact subset $K$ of a space $X$ is proximally $\infty$-connected if, for each $\varepsilon>0$, there is $0<\delta \leq \varepsilon$ such that the inclusion $N_{\delta}(K) \hookrightarrow N_{\varepsilon}(K)$ induces the trivial homomorphism

$$
\pi_{n}\left(N_{\delta}(K)\right) \rightarrow \pi_{n}\left(N_{\varepsilon}(K)\right)
$$

for any $n \geq 0$ (we suppress the base points from the notations since they are not necessary).

Rem ark 2.2. In [5] a whole list of examples of proximally $\infty$-connected sets is given. In particular any $R_{\delta}$-set (i.e. the intersection of a decreasing sequence of compact AR's, see [8]) lying in an ANR space is proximally $\infty$-connected. This example will be important for us in the last section.

\section{Definition 2.3 .}

(i) (comp. [5]) If $\varphi: X \rightarrow Y$ is a set-valued map, then we say that $\varphi$ belongs to the class $J$ if, for any $x \in X, \varphi(x)$ is proximally $\infty$-connected.

(ii) A set-valued map $\varphi: X \rightarrow Y$ is said to be decomposable, provided there is a factorisation

$$
D_{\varphi}: X=X_{0} \stackrel{\varphi_{1}}{\longrightarrow} X_{1} \stackrel{\varphi_{2}}{\longrightarrow} \cdots \stackrel{\varphi_{n}}{\longrightarrow} X_{n}=Y,
$$

$\left(n=n(\varphi)\right.$ depends on $\varphi$ ) where $\varphi_{i}$ are $J$-maps, $1 \leq i \leq n, X_{0}, \ldots, X_{n}$ are ANR's such that $\varphi=\varphi_{n} \circ \cdots \circ \varphi_{1}$. In this case $D_{\varphi}$ is a decomposition of $\varphi$.

Now let $\varphi: X \rightarrow X$ be compact, decomposable with a decomposition

$$
D_{\varphi}: X=X_{0} \stackrel{\varphi_{1}}{\longrightarrow} X_{1} \stackrel{\varphi_{2}}{\longrightarrow} \cdots \stackrel{\varphi_{n}}{\longrightarrow} X_{n}=X
$$

and $W$ be an open subset of $X$ such that $\operatorname{Fix}(\varphi) \cap \partial W=\emptyset$. There exists a fixed point 
index

$$
\text { Ind }\left(X, D_{\varphi}, W\right) \in \mathbb{Z}
$$

for the decomposition $D_{\varphi}$ of $\varphi$ on $W$.

We have indicated by the notation, that Ind depends not only on $\varphi$ but also on the decomposition $D_{\varphi}$ involved. On the other hand we have the following result:

Proposition 2.4 Let $\varphi$ be compact, decomposable with decomposition $D_{\varphi}$ (see (3)), $W$ an open subset of $X$ and $\operatorname{Fix}(\varphi) \cap \partial W=\emptyset$. Let

$$
D^{\prime}: X=X_{0}^{\prime} \stackrel{\varphi_{1}^{\prime}}{\longrightarrow} X_{1}^{\prime} \stackrel{\varphi_{2}^{\prime}}{\longrightarrow} \cdots \stackrel{\varphi_{n}^{\prime}}{\longrightarrow} X_{n}^{\prime}=X
$$

be such that for each $1 \leq i \leq n$, there is $h_{i}: X_{i} \rightarrow X_{i}^{\prime}$ with $h_{0}=h_{n}=\operatorname{id}_{X}$ and the diagram

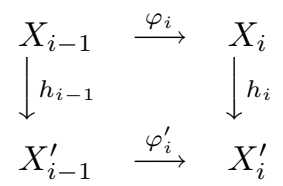

commutes (i.e. $\left.\varphi_{i}^{\prime} \circ h_{i-1}=h_{i} \circ \varphi_{i}\right)$ for $1 \leq i \leq n$. Then

$$
\text { Ind }\left(X, D_{\varphi}, W\right)=\operatorname{Ind}\left(X, D^{\prime}, W\right) \text {. }
$$

If decompositions are as in the proposition we say that $D^{\prime}$ dominates over $D_{\varphi}$ (written $\left.D^{\prime}>D_{\varphi}\right)$.

Below we shall state the properties of the fixed point index, which we use in the sequel. First we need the notion of homotopy for decompositions.

Definition 2.5. Let $\varphi, \psi: X \rightarrow Y$ be decomposable with decompositions $D_{\varphi}$ (see (2)) and

$$
D_{\psi}: X=X_{0}^{\prime} \stackrel{\psi_{1}}{\longrightarrow} X_{1}^{\prime} \stackrel{\psi_{2}}{\longrightarrow} \cdots \stackrel{\psi_{m}}{\longrightarrow} X_{m}^{\prime}=Y .
$$

We say that the decompositions $D_{\varphi}$ and $D_{\psi}$ are homotopic if $n=m, X_{i}=X_{i}^{\prime}$, and there is a map $\chi_{i} \in J\left(X_{i-1} \times I, X_{i}\right)$ with $\chi_{i}(\cdot, 0)=\varphi_{i}, \chi_{i}(\cdot, 1)=\psi_{i}, 1 \leq i \leq n$. The set-valued map $\chi: X \times I \rightarrow Y$ given by

$$
\chi(x, t):=\chi_{n}\left(\bar{\chi}_{n-1}\left(\ldots \bar{\chi}_{1}(x, t) \ldots\right),\right.
$$

where $\bar{\chi}_{i}(x, t)=\chi_{i}(x, t) \times\{t\}$ for $x \in X_{i-1}, t \in I, 1 \leq i \leq n-1$, is called a homotopy (observe that $\chi(\cdot, 0)=\varphi, \chi(\cdot, 1)=\psi$ ). We say that the decompositions $D_{\varphi}, D_{\psi}$ are compactly homotopic, if $\chi$ is compact.

TheOREM 2.6. Let $\varphi$ be compact, decomposable with decomposition (3), W an open subset of $X$ and Fix $(\varphi) \cap \partial W=\emptyset$.

(i) (Existence) If Ind $\left(X, D_{\varphi}, W\right) \neq 0$ then $\operatorname{Fix}(\varphi) \cap W \neq \emptyset$.

(ii) (Additivity) If $\operatorname{Fix}(\varphi) \cap W \subset \bigcup_{j=1}^{k} W_{j}$, where $W_{j}, 1 \leq j \leq k$, are disjoint open 
subsets in $W$, then

$$
\operatorname{Ind}\left(X, D_{\varphi}, W\right)=\sum_{j=1}^{k} \operatorname{Ind}\left(X, D_{\varphi}, W_{j}\right) .
$$

(iii) (Homotopy) If $\psi$ is compact, decomposable with a decomposition

$$
D_{\psi}: X=X_{0}^{\prime} \stackrel{\psi_{1}}{\longrightarrow} X_{1}^{\prime} \stackrel{\psi_{2}}{\longrightarrow} \cdots \stackrel{\psi_{m}}{\longrightarrow} X_{m}^{\prime}=X
$$

compactly homotopic to $D_{\varphi}$ and the homotopy $\chi: X \times I \rightarrow X$ is such that $x \notin$ $\chi(x, t)$ for $x \in \partial W, t \in I$, then

$$
\text { Ind }\left(X, D_{\varphi}, W\right)=\operatorname{Ind}\left(X, D_{\psi}, W\right) \text {. }
$$

(iv) (Units) If $\varphi$ is constant, i.e. for any $x \in X, \varphi(x)=T$ and $T \subset X$ is proximally $\infty$-connected, then

$$
\text { Ind }\left(X, D_{\varphi}, W\right)= \begin{cases}1 & \text { if } W \cap T \neq \emptyset \\ 0 & \text { if } W \cap T=\emptyset\end{cases}
$$

COROllary 2.7. Let $X$ be a closed convex subset of a normed space (and hence an ANR), $W$ an open subset of $X$ and $\varphi, \psi: X \rightarrow X$ compact, decomposable with decompositions $D_{\varphi}, D_{\psi}$ (see (3), (4)). Let the set-valued map $\lambda: X \times I \rightarrow X$ given by

$$
\lambda(x, t):=(1-t) \varphi(x)+t \psi(x)
$$

be such that

$$
x \notin \lambda(x, t)
$$

for $x \in \partial W, t \in I$. Then $\operatorname{Ind}\left(X, D_{\varphi}, W\right)=\operatorname{Ind}\left(X, D_{\psi}, W\right)$.

Observe that $\lambda$ does not establish a homotopy of the decompositions $D_{\varphi}, D_{\psi}$ and thus (2.6)(iii) can not be applied directly.

Proof. We may assume without loss of generality $m \leq n$. Define $X_{m+1}^{\prime}, \ldots, X_{n}^{\prime}:=$ $X, \psi_{m+1}, \ldots, \psi_{n}:=i d_{X}$. Consider the decompositions

$$
\begin{aligned}
& D_{1}: X \stackrel{d}{\longrightarrow} X \times X \stackrel{\varphi_{1} \times \psi_{1}}{\longrightarrow} X_{1} \times X_{1}^{\prime} \stackrel{\varphi_{2} \times \psi_{2}}{\longrightarrow} \cdots \stackrel{\varphi_{n} \times \psi_{n}}{\longrightarrow} X \times X \stackrel{p_{0}}{\longrightarrow} X, \\
& D_{2}: X \stackrel{d}{\longrightarrow} X \times X \stackrel{\varphi_{1} \times \psi_{1}}{\longrightarrow} X_{1} \times X_{1}^{\prime} \stackrel{\varphi_{2} \times \psi_{2}}{\longrightarrow} \cdots \stackrel{\varphi_{n} \times \psi_{n}}{\longrightarrow} X \times X \stackrel{q_{0}}{\longrightarrow} X,
\end{aligned}
$$

where $d(x):=(x, x), p_{0}(x, y):=x, q_{0}(x, y):=y$. The $J$-maps

$$
\begin{aligned}
D & : X \times I \rightarrow X \times X, D(x, t):=d(x), \\
\chi_{i} & : X_{i-1} \times X_{i-1}^{\prime} \times I \rightarrow X_{i} \times X_{i}^{\prime}, \chi_{i}(x, y, t):=\left(\varphi_{i} \times \psi_{i}\right)(x, y), 1 \leq i \leq n, \\
\chi_{n+1} & : X \times X \times I \rightarrow X, \chi_{n+1}(x, y, t):=(1-t) x+t y .
\end{aligned}
$$

show that $D_{1}$ and $D_{2}$ are homotopic in the sense of definition (2.5). But since

$$
\lambda(x, t)=\chi_{n+1}\left(\overline { \chi } _ { n } \left(\ldots \bar{\chi}_{1}(\bar{D}(x, t) \ldots),\right.\right.
$$

and $\lambda$ clearly is compact, it follows from (5) and (2.6)(iii) that

$$
\text { Ind }\left(X, D_{1}, W\right)=\operatorname{Ind}\left(X, D_{2}, W\right) \text {. }
$$


The proof is finished if we show

$$
\begin{aligned}
& \text { Ind }\left(X, D_{\varphi}, W\right)=\operatorname{Ind}\left(X, D_{1}, W\right), \\
& \text { Ind }\left(X, D_{\psi}, W\right)=\operatorname{Ind}\left(X, D_{2}, W\right) .
\end{aligned}
$$

To this end, define maps $p_{i}: X_{i} \times X_{i}^{\prime} \rightarrow X_{i}, p_{i}(x, y):=x$ for $1 \leq i \leq n$. Since $p_{0} \circ d=i d_{X}$, $p_{i} \circ\left(\varphi_{i} \times \psi_{i}\right)=\varphi_{i} \circ p_{i-1}$ and $p_{0}=i d_{X} \circ p_{n}$ it follows that $D_{\varphi}>D_{1}\left(D_{\psi}>D_{2}\right.$ is shown analogously). Hence, the above equalities follow from (2.4).

Corollary 2.8. Let $X$ be a closed convex subset of a normed space, $\varphi: X \rightarrow X$ compact, decomposable with decomposition $D_{\varphi}$. Then $\operatorname{Ind}\left(X, D_{\varphi}, X\right)=1$.

3. Multiple positive fixed points on ordered normed spaces. The purpose of this section is to prove some of the abstract results which will be applied in the problem of periodic solutions of differential inclusions.

We recall that a closed convex subset $K$ of a normed space $E$ is called a cone if $t x \in K$ for each $x \in K$ and $t \geq 0$ and if $x \in K$ and $-x \in K$, then $x=0$. A cone $K$ induces a partial ordering $\leq$ in $E$ by $x \leq y$ if and only if $y-x \in K$.

By a convex (resp. concave) functional $a$ (resp. $b$ ) on $K$ we mean a mapping $a: K \rightarrow$ $\mathbb{R}($ resp. $b: K \rightarrow \mathbb{R}$ ) such that

$$
\begin{aligned}
a(t x+(1-t) y) \leq t a(x)+(1-t) a(y) & \text { for } x, y \in K, t \in I \\
(\text { resp. } b(t x+(1-t) y) \geq t b(x)+(1-t) b(y) & \text { for } x, y \in K, t \in I) .
\end{aligned}
$$

Lemma 3.1. Let $\varphi: K \rightarrow K$ be a compact, decomposable map with the decomposition

$$
D_{\varphi}: K=X_{0} \stackrel{\varphi_{1}}{\longrightarrow} X_{1} \stackrel{\varphi_{2}}{\longrightarrow} \cdots \stackrel{\varphi_{n}}{\longrightarrow} X_{n}=K \text {. }
$$

Let $a, b$ be convex, concave functionals on $K$ respectively and $r, s$ numbers such that

(a) the set $U:=\{x \in K \mid a(x)<r, b(x)>s\}$ is nonempty,

(b) $a(x)=r, b(x) \geq s, y \in \varphi(x) \Rightarrow a(y)<r$,

(c) $b(x)=s, a(x) \leq r, y \in \varphi(x) \Rightarrow b(y)>s$.

Then Ind $\left(K, D_{\varphi}, U\right)=1$.

Proof. The set $U$ is obviously open. Since $x \in \partial U$ implies either $a(x)=r$ and $b(x) \geq s$ or $a(x) \leq r$ and $b(x)=s$, we see, by (b) and (c), that $x \notin \varphi(x)$. It follows that Ind $\left(K, D_{\varphi}, U\right)$ is defined.

Take a point $x_{0} \in U$ and the obvious decomposition $D_{x_{0}}: K \stackrel{x_{0}}{\longrightarrow} K$ of the constant map sending each point to $x_{0}$. Consider

$$
\lambda: K \times I \rightarrow K, \lambda(x, t):=(1-t) \varphi(x)+t x_{0} .
$$

Suppose that $x \in \lambda(x, t)$ for some $x \in \partial U, t \in I$. Then there is $y \in \varphi(x)$ such that $x=(1-t) y+t x_{0}$. Now, if $a(x)=r$ and $b(x) \geq s$ then

$$
r=a(x)=a\left((1-t) y+t x_{0}\right) \leq(1-t) a(y)+t a\left(x_{0}\right)<r,
$$

by convexity of $a$ and (b). If on the other hand $a(x) \leq r$ and $b(x)=s$, then

$$
s=b(x)=b\left((1-t) y+t x_{0}\right) \geq(1-t) b(y)+t b\left(x_{0}\right)>s .
$$


Hence in both cases we obtain a contradiction. Thus we have shown $x \notin \lambda(x, t)$ for each $(x, t) \in \partial U \times I$ and by $(2.7)$ we obtain,

$$
\text { Ind }\left(K, D_{\varphi}, U\right)=\operatorname{Ind}\left(K, D_{x_{0}}, U\right) \text {. }
$$

Finally using property $(2.6)(\mathrm{iv})$ we see that $\operatorname{Ind}\left(K, D_{x_{0}}, U\right)=1$.

THEOREM 3.2. Let $\varphi$ be as above and $m \geq 2$. Let $a_{1}, \ldots, a_{m}$ be convex functionals, $b_{1}, \ldots, b_{m}$ concave functionals on $K$ and numbers $r_{1}, \ldots, r_{m}, s_{1}, \ldots, s_{m}$ be given such that:

(a) $a_{m}(x) \leq a_{m-1}(x) \leq \ldots \leq a_{1}(x), b_{m}(x) \leq b_{m-1}(x) \leq \ldots \leq b_{1}(x)$ for $x \in K$,

(b) $r_{1} \leq r_{2} \leq \ldots \leq r_{m}, s_{1} \leq s_{2} \leq \ldots \leq s_{m}$,

(c) $b_{i}(x) \leq a_{i-1}(x)$ for $x \in K, r_{i-1} \leq s_{i}, 2 \leq i \leq m$,

(d) the sets $U_{i}:=\left\{x \in K \mid a_{i}(x)<r_{i}, b_{i}(x)>s_{i}\right\}, 1 \leq i \leq m$, are nonempty,

(e) $a_{i}(x)=r_{i}, b_{i}(x) \geq s_{i}, y \in \varphi(x) \Rightarrow a_{i}(y)<r_{i}, 1 \leq i \leq m$,

(f) $b_{i}(x)=s_{i}, a_{i}(x) \leq r_{i}, y \in \varphi(x) \Rightarrow b_{i}(y)>s_{i}, 1 \leq i \leq m$.

Then $\varphi$ has at least $m+1$ fixed points.

Proof. By lemma (3.1), using (d), (e) and (f) above, we have

$$
\text { Ind }\left(K, D_{\varphi}, U_{i}\right)=1, \quad 1 \leq i \leq m \text {. }
$$

Thus, by the existence property (2.6)(i) of Ind the existence of $m$ fixed points is established, if we show that the $U_{i}$ 's are disjoint. Let $U_{i}$ be given. Then, if $1 \leq j \leq i-1$ we see that if $x \in U_{j}$ then $a_{i-1}(x) \leq a_{j}(x)<r_{j} \leq r_{i-1}$ by (a) and (b). But, by (c), we have that $b_{i}(x) \leq a_{i-1}(x)<r_{i-1} \leq s_{i}$. It follows that if $x \in U_{j}$ then $b_{i}(x)<s_{i}$ and, hence $x \notin U_{i}$. If $i+1 \leq j \leq m$ we conclude the same from $b_{i+1}(x) \geq b_{j}(x)>s_{j} \geq s_{i+1}$ and $r_{i} \leq s_{i+1}<b_{i+1}(x) \leq a_{i}(x)$.

Now consider $U:=K \backslash\left(\overline{U_{1} \cup \ldots \cup U_{m}}\right)$. Then $U$ is an open set and $U, U_{1}, \ldots, U_{n}$ are disjoint. Moreover, since $K \backslash\left(U \cup U_{1} \cup \ldots \cup U_{m}\right)=\partial U_{1} \cup \ldots \cup \partial U_{m}$ and there are no fixed points on $\partial U_{i}, 1 \leq i \leq m$, we see that $\operatorname{Fix}(\varphi) \subset U \cup U_{1} \cup \ldots \cup U_{m}$. Hence, by additivity (2.6)(ii), and (2.8) we obtain

$$
1=\operatorname{Ind}\left(K, D_{\varphi}, K\right)=\operatorname{Ind}\left(K, D_{\varphi}, U\right)+\sum_{i=1}^{m} \operatorname{Ind}\left(K, D_{\varphi}, U_{i}\right) .
$$

By virtue of (6),

$$
1-m=\operatorname{Ind}\left(K, D_{\varphi}, U\right)
$$

and since $m \geq 2$ we see that

$$
\text { Ind }\left(K, D_{\varphi}, U\right) \neq 0 \text {, }
$$

so that $\varphi$ has another fixed point in $U$. It follows that $\varphi$ has at least $m+1$ fixed points. $\square$

By strengthening the assumptions a bit we obtain additional intermediate fixed points.

TheOREm 3.3. Let all the conditions of theorem (3.2) be fulfilled, but replace (e) and (f) $b y$

(e') $a_{i}(x)=r_{i}, y \in \varphi(x) \Rightarrow a_{i}(y)<r_{i}, 1 \leq i \leq m$,

(f') $b_{i}(x)=s_{i}, a_{m}(x) \leq r_{m}, y \in \varphi(x) \Rightarrow b_{i}(y)>s_{i}, 1 \leq i \leq m$. 
Then $\varphi$ has at least $2 m-1$ fixed points.

Proof. Following the first part of the proof of theorem (3.2) one sees that

$$
\text { Ind }\left(K, D_{\varphi}, U_{i}\right)=1, \quad 1 \leq i \leq m,
$$

and the sets $U_{i}, 1 \leq i \leq m$, are disjoint. We obtain $m$ fixed points. Now define open sets

$$
\begin{aligned}
V_{i} & :=\left\{x \in K \mid a_{i}(x)<r_{i}, b_{i-1}(x)>s_{i-1}\right\}, & \\
W_{i} & :=V_{i} \cap\left(K \backslash\left(\overline{U_{i} \cup U_{i-1}}\right)\right) & \text { for } 2 \leq i \leq m .
\end{aligned}
$$

By lemma (3.1) it follows that

$$
\text { Ind }\left(K, D_{\varphi}, V_{i}\right)=1 \quad \text { for } 2 \leq i \leq m .
$$

Since $V_{i} \backslash\left(U_{i-1} \cup U_{i} \cup W_{i}\right) \subset \partial U_{i} \cup \partial U_{i-1}$ we have

$$
\operatorname{Fix}(\varphi) \cap V_{i} \subset U_{i-1} \cup U_{i} \cup W_{i} .
$$

Of course the sets $U_{i-1}, U_{i}, W_{i}$ are disjoint, so that we obtain, by (8) and (2.6)(ii),

$$
\operatorname{Ind}\left(K, D_{\varphi}, U_{i-1}\right)+\operatorname{Ind}\left(K, D_{\varphi}, U_{i}\right)+\operatorname{Ind}\left(K, D_{\varphi}, W_{i}\right)=\operatorname{Ind}\left(K, D_{\varphi}, V_{i}\right)=1 .
$$

Using (7), it follows that

$$
\text { Ind }\left(K, D_{\varphi}, W_{i}\right)=-1
$$

The sets $W_{i}, 2 \leq i \leq m$ are disjoint: $W_{i-1} \cap W_{i}=\emptyset$ follows since $x \in W_{i-1} \cap W_{i}$ would imply $x \in U_{i-1}$, which is impossible. It remains to prove $W_{i} \cap W_{j}=\emptyset$ if $j=2, \ldots, i-2, i+$ $2, \ldots, m$. This follows if $2 \leq j \leq i-2$ from $b_{i-1}(x) \leq a_{i-2}(x) \leq a_{j}(x)<r_{j} \leq r_{i-2} \leq s_{i-1}$ and, in case $i+2 \leq j \leq m$ from $a_{i}(x) \geq b_{i+1}(x) \geq b_{j-1}(x)>s_{j-1} \geq s_{i+1} \geq r_{i}$. Hence, we see that $\varphi$ has $m-1$ fixed points in $W_{2}, \ldots, W_{m}$. The proof is finished if we show

$$
W_{i} \cap U_{j}=\emptyset \quad \text { for } 1 \leq j \leq m \text {. }
$$

If $j=i, i-1$ this is obvious, so let $1 \leq j \leq i-2$ : In this case we conclude from $b_{i-1}(x) \leq a_{i-2}(x) \leq a_{j}(x)<r_{j} \leq r_{i-2} \leq s_{i-1}$ and if $i+1 \leq j \leq m$ consider $a_{i}(x) \geq$ $b_{i+1}(x) \geq b_{j}(x)>s_{j} \geq s_{i+1} \geq r_{i}$.

COROLlary 3.4. If all conditions of the above theorem are fulfilled, but instead of $\varphi$ compact, let $\varphi$ be completely continuous. Moreover let

$$
\lim _{\|x\| \rightarrow \infty} a_{m}(x)=\infty .
$$

Then $\varphi$ has at least $2 m-1$ fixed points.

Pr o of. The set $A:=\left\{x \in K \mid a_{m}(x) \leq r_{m}\right\}$ is closed, bounded, convex and therefore an AR. Hence we can define a compact, decomposable map $\varphi^{\prime}: K \rightarrow K$ such that $\varphi^{\prime}(x)=\varphi(x)$ for $x \in A$. Applying the theorem gives $2 m-1$ fixed points for $\varphi^{\prime}$ and all the fixed points are in $A$. Thus we have also $2 m-1$ fixed points of $\varphi$.

4. Multiple periodic solutions of differential inclusions. Let $K$ be a cone in $\mathbb{R}^{n}$ and $\Phi:[0, T] \times K \rightarrow \mathbb{R}^{n}$ a set-valued map.

Given a point $x_{0} \in K$ we consider the initial value problem

$$
\left\{\begin{array}{l}
x^{\prime}(t) \in \Phi(t, x(t)) \\
x(0)=x_{0}
\end{array}\right.
$$


An absolutely continuous map $x:[0, T] \rightarrow K$ is a solution of $(9)$ if $x^{\prime}(t) \in \Phi(t, x(t))$ for a.e. $t \in[0, T]$ and $x(0)=x_{0}$. We denote by

$$
S_{\Phi}\left(x_{0}\right):=\{x:[0, T] \rightarrow K \mid x \text { is a solution of }(9)\}
$$

the set of all solutions.

In order to guarantee the existence of solutions of (9) we shall assume the following boundary condition:

$$
\Phi(t, x) \cap T_{K}(x) \neq \emptyset \quad \text { for each } t \in[0, T], x \in K,
$$

where $T_{K}(x)$ is the Bouligand cone to $K$ at the point $x \in K$, i.e.

$$
T_{K}(x):=\left\{y \in \mathbb{R}^{n} \mid \liminf _{h \rightarrow+0} \frac{\operatorname{dist}(x+h y, K)}{h}=0\right\} .
$$

We have the following result (comp. [4]):

Theorem 4.1. Let $\Phi:[0, T] \times K \rightarrow \mathbb{R}^{n}$ be a convex valued, bounded map such that (10) holds. Then

$$
S_{\Phi}: K \rightarrow C([0, T], K)
$$

is a $J$-map $\left(\right.$ by $C([0, T], K)$ we denote the subspace of $C\left([0, T], \mathbb{R}^{n}\right)$ of mappings $[0, T] \rightarrow$ $K)$.

Proof. Let $x_{0} \in K$. It follows from the results in [11] (comp. [6]) that $S_{\Phi}\left(x_{0}\right)$ is an $R_{\delta}$-set. Since $C([0, T], K)$ - a closed convex subset of $C\left([0, T], \mathbb{R}^{n}\right)$ - is an ANR, (2.2) implies that $S_{\Phi}\left(x_{0}\right) \subset C([0, T], K)$ is proximally $\infty$-connected. Finally, using standard arguments (comp. [2], p.79) we see that $S_{\Phi}$ is upper semi-continuous.

Consider next the periodic problem

$$
\left\{\begin{array}{l}
x^{\prime}(t) \in \Phi(t, x(t)) \\
x(0)=x(T)
\end{array}\right.
$$

Let $e_{T}: C([0, T], K) \rightarrow K$ be the evaluation map $e_{T}(x):=x(T)$ in $T$ and consider

$$
K \stackrel{S_{\Phi}}{\longrightarrow} C([0, T], K) \stackrel{e_{T}}{\longrightarrow} K .
$$

This decomposition defines a set-valued map $P_{\Phi}:=e_{T} \circ S_{\Phi} ; P_{\Phi}$ is called the Poincaré translation operator. It is evident that problem (11) is equivalent to the existence of a fixed point of $P_{\Phi}$.

In order to apply the results of the third section we have to control the flow of the considered dynamical system.

Lemma 4.2. Let $\Phi:[0, T] \times K \rightarrow \mathbb{R}^{n}$ be a convex valued, bounded map, a $: \mathbb{R}^{n} \rightarrow \mathbb{R}$ continuously differentiable and let $r$ be a number such that

$$
\langle\operatorname{grad} a(x), y\rangle<0 \quad \text { for } t \in[0, T], a(x)=r \text { and } y \in \Phi(t, x) .
$$

If $a\left(x_{0}\right) \leq r$ and $x \in S_{\Phi}\left(x_{0}\right)$ then $a(x(t))<r$ for $t \in(0, T]$.

Proof. Let $g:[0, T] \rightarrow \mathbb{R}, g(t):=a(x(t))$. Then $g^{\prime}(t)=\left\langle\operatorname{grad} a(x(t)), x^{\prime}(t)\right\rangle$ a.e. Now, assume that $t>0$ is the smallest real such that $g(t)=r$. But, using (12) and the upper semi-continuity of $\Phi$, we see that $g^{\prime}(s)<0$ a.e. on some $\varepsilon$-neighborhood of $t$ in 
$[0, T]$. It follows for $t^{\prime}<t,\left|t-t^{\prime}\right|<\varepsilon$, that $g\left(t^{\prime}\right)=g(t)-\int_{t^{\prime}}^{t} g^{\prime}(s) d s>g(t)=r$ and thus we obtain a contradiction.

Corollary 4.3. Let $b: \mathbb{R}^{n} \rightarrow \mathbb{R}$ be continuously differentiable and let $s$ be a number such that

$$
\langle\operatorname{grad} b(x), y\rangle>0 \quad \text { for } t \in[0, T], b(x)=s \text { and } y \in \Phi(t, x) \text {. }
$$

If $b\left(x_{0}\right) \geq s$ and $x \in S_{\Phi}\left(x_{0}\right)$ then $b(x(t))>s$ for $t \in(0, T]$.

We are now able to state our main results. We will give two versions of theorems where multiple periodic solutions can be obtained.

TheOREM 4.4. Let $\Phi:[0, T] \times K \rightarrow \mathbb{R}^{n}$ be a convex valued, bounded map such that (10) holds. Let $a_{1}, \ldots, a_{m}$ be continuously differentiable convex functionals, $b_{1}, \ldots, b_{m}$ continuously differentiable concave functionals on $K$ and numbers $r_{1}, \ldots, r_{m}, s_{1}, \ldots, s_{m}$ be given such that:

(a) $a_{m}(x) \leq a_{m-1}(x) \leq \ldots \leq a_{1}(x), b_{m}(x) \leq b_{m-1}(x) \leq \ldots \leq b_{1}(x)$ for $x \in K$,

(b) $r_{1} \leq r_{2} \leq \ldots \leq r_{m}, s_{1} \leq s_{2} \leq \ldots \leq s_{m}$,

(c) $b_{i}(x) \leq a_{i-1}(x)$ for $x \in K, r_{i-1} \leq s_{i}, 2 \leq i \leq m$,

(d) the sets $U_{i}:=\left\{x \in K \mid a_{i}(x)<r_{i}, b_{i}(x)>s_{i}\right\}, 1 \leq i \leq m$, are nonempty,

(e) $\lim _{\|x\| \rightarrow \infty} a_{m}(x)=\infty$,

(f) $\left\langle\operatorname{grad} a_{i}(x), y\right\rangle<0$ for $t \in[0, T], a_{i}(x)=r_{i}$ and $y \in \Phi(t, x), 1 \leq i \leq m$,

(g) $\left\langle\operatorname{grad} b_{i}(x), y\right\rangle>0$ for $t \in[0, T], b_{i}(x)=s_{i}$ and $y \in \Phi(t, x), 1 \leq i \leq m$.

Then $x^{\prime} \in \Phi(t, x)$ has at least $2 m-1$ periodic solutions.

Pr o of. The Poincaré translation operator $P_{\Phi}$ is decomposable and, by finite-dimensionality, completely continuous. Considering (a)-(g) above, and using (4.2) and (4.3), we see that all the conditions of Corollary (3.4) are satisfied. Hence we have $2 m-1$ fixed points of $P_{\Phi}$.

TheOrem 4.5. Let $\Phi:[0, T] \times K \rightarrow \mathbb{R}^{n}$ be a convex valued, bounded map such that (10) holds. Let $a, b: \mathbb{R}^{n} \rightarrow \mathbb{R}$ be continuously differentiable, convex, concave functionals on $K$ respectively and numbers $r, s$ such that

(a) $\|x\| \leq a(x), b(x) \leq a(x)$ for $x \in K$ and $r \leq s$,

(b) $\{x \in K \mid a(x)<r\} \neq \emptyset$,

(c) $\lim _{\|x\| \rightarrow \infty} b(x)=\infty$,

(d) $\langle\operatorname{grad} a(x), y\rangle<0$ for $t \in[0, T], a(x)=r$ and $y \in \Phi(t, x)$,

(e) $\langle\operatorname{grad} b(x), y\rangle>0$ for $t \in[0, T], b(x)=s$ and $y \in \Phi(t, x)$.

Then $x^{\prime} \in \Phi(t, x)$ has at least two periodic solutions. Particularly, there exists a nonzero periodic solution.

P r o of. We apply theorem (3.3) for $m=2$. By condition (c) there is $c>r$ such that $\|x\| \geq c$ implies $b(x)>s$. Since $\Phi$ is bounded there is $r^{\prime}$ such that for any $x_{0},\left\|x_{0}\right\| \leq c$ it follows that if $x \in S_{\Phi}\left(x_{0}\right)$ then $\|x(t)\|<r^{\prime}$ for $t \in[0, T]$. Define $f: K \rightarrow K$,

$$
f(x):= \begin{cases}c x /\|x\| & \text { if }\|x\| \geq c, \\ x & \text { if }\|x\| \leq c\end{cases}
$$


and consider the decomposition

$$
K \stackrel{S_{\Phi} \circ f}{\longrightarrow} C([0, T], K) \stackrel{e_{T}}{\longrightarrow} K
$$

of the map $e_{T} \circ S_{\Phi} \circ f=P_{\Phi} \circ f$. Define $a_{1}:=a, a_{2}:=\|\cdot\|, b_{2}:=b, r_{1}:=r, r_{2}:=r^{\prime}$ and $s_{2}:=s$. One easily sees that the conditions of (3.3) are fulfilled, so that we obtain three fixed points of $P_{\Phi} \circ f$, where in each one of the disjoint sets (with the notation of (3.3)) $U_{1}, U_{2}, W_{2}$ lies at least one fixed point. But, since $U_{1} \cup W_{2} \subset\{x \in K \mid\|x\| \leq c\}$, we see that $f$ has no influence on the fixed points lying in $U_{1}$ or $W_{2}$ so that we indeed obtain at least two fixed points of $P_{\Phi}$.

Acknowledgment. I would like to thank Dr. W. Kryszewski for raising my interest in the questions considered in the paper.

\section{References}

[1] R. Bader and W. Kryszewski, Fixed-point index for compositions of set-valued maps with proximally $\infty$-connected values on arbitrary ANR's, Set-Valued Analysis 2 (1994), 459-480.

[2] K. Deimling, Multivalued differential equations, de Gruyter, 1992.

[3] J. Dugundji, Modified Vietoris theorems for homotopy, Fund. Math. 66 (1970), 223235.

[4] L. Górniewicz, Topological approach to differential inclusions, in: Topological Methods in Differential Equations and Inclusions, A. Granas, M. Frigon (eds.), NATO ASI Series C 472, Kluwer Academic Publ., 1995, 129-190.

[5] L. Górniewicz, A. Granas and W. Kryszewski, On the homotopy method in the fixed point index theory of multivalued mappings of compact ANR's, J. Math. Anal. Appl. 161 (1991), 457-473.

[6] L. Górniewicz, P. Nistri and P. Zecca, Control problems on closed subsets of $\mathbb{R}^{n}$ via feedback controls, Topol. Methods Nonlinear Anal., 2 (1993), 163-178.

[7] L. Górniewicz and S. Plaskacz, Periodic solutions of differential inclusions in $\mathbb{R}^{n}$, Bollettino U.M.I., (7), 7-A (1993), 409-420.

[8] D. M. Hyman, On decreasing sequences of compact absolute retracts, Fund. Math., 64 (1969), 91-97.

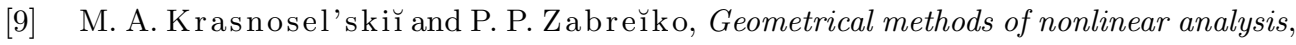
Springer Verlag, 1984

[10] R. W. Leggett and L. R. Williams, Multiple positive fixed points of nonlinear operators on ordered Banach spaces, Indiana Univ. Math. J., 28 (1979), 673-689.

[11] S. Plaskacz, On the solution sets for differential inclusions, Bollettino U.M.I., (7), 6-A (1992), 387-394. 